\title{
The globular cluster tidal radii $\dagger$
}

\author{
Z. Y. Wu \\ National Astronomical Observatories, Chinese Academy of Sciences, Beijing 100012, China \\ email: zywu@bao.ac.cn
}

\begin{abstract}
The orbits and theoretical tidal radii for a sample of 45 Galactic globular clusters are calculated. The orbital phase dependence between the theoretical and observed tidal radii is noted.
\end{abstract}

Keywords. Galaxy: kinematics and dynamics, globular clusters: general

\section{Introduction}

It has long been recognized that globular clusters must have a finite edge imposed by the Galactic tidal field. Most observed surface density profiles of globular clusters can be fitted by the King model (King 1966), while the edge of a globular cluster has traditionally been estimated using the model-predicted value of the King's tidal radius, $r_{t}$. The classical theory has pointed out that the observed tidal radii of globular clusters were obtained when the Galactic tidal force on the clusters was the strongest, that is at the perigalactic position (Hoerner 1957 and King 1962). The classical tidal radius theory does not include the internal dynamical processes: the two-body relaxation, and the energy input caused by shocks when clusters cross through the disk or the central bulge (Wu et al. 2003). If the tidal radius of a globular cluster is imposed at the perigalactic postion, then owing to internal and external dynamical effects, this tidal radius will expand along the orbit of a cluster, i.e. there will be an orbital phase dependence between the theoretical tidal radii at the perigalactic position and the presently observed tidal radii.

\section{Orbits and tidal radii of globular clusters}

$\mathrm{Wu}$, et al. (2004) calculated the orbits of 45 globular clusters with a simple Galactic mass model: $M_{g}\left(R_{g}\right)=V_{c}^{2} R_{g} / G$, where $\mathrm{G}$ is the constant of gravitation, $V_{c}=220.0 \mathrm{~km}$ $\mathrm{s}^{-1}$ the circle velocity, and $R_{g}$ the Galactocentric distance. The theoretical tidal radius of a globular cluster $r_{t e}$ is determined using the formula of Innanen (1983):

$$
r_{t e}=\frac{2}{3}\left[1-\ln \left(R_{p} / A\right)\right]^{-1 / 3}\left(\frac{m}{2 M_{p}}\right)^{1 / 3} R_{p}
$$

where $R_{p}$ is the perigalactic distance of the cluster, $m$ is the mass of the cluster, $M_{p}=$ $M_{g}\left(R_{p}\right)$ is the mass of the Galaxy within $R_{p}$, and $A$ is a distance taken as the radius of a circular orbit which has the same total orbital energy for any given cluster orbit. We use the last perigalactic crossing to compute the theoretical tidal radius $r_{t e}$. The present observed tidal radii of the clusters mostly are taken from the compilation of Trager et al. (1993). Panel a of Fig. 1 shows that, for most of the clusters, the theoretical values are small compared to the observed ones. Thus, the theoretical tidal radius calculated at the perigalactic distance gives an underestimated value of the observed one. The theoretical

$\dagger$ This work has been supported in part by the project No.10778720 of National Natural Science Foundation of China. 

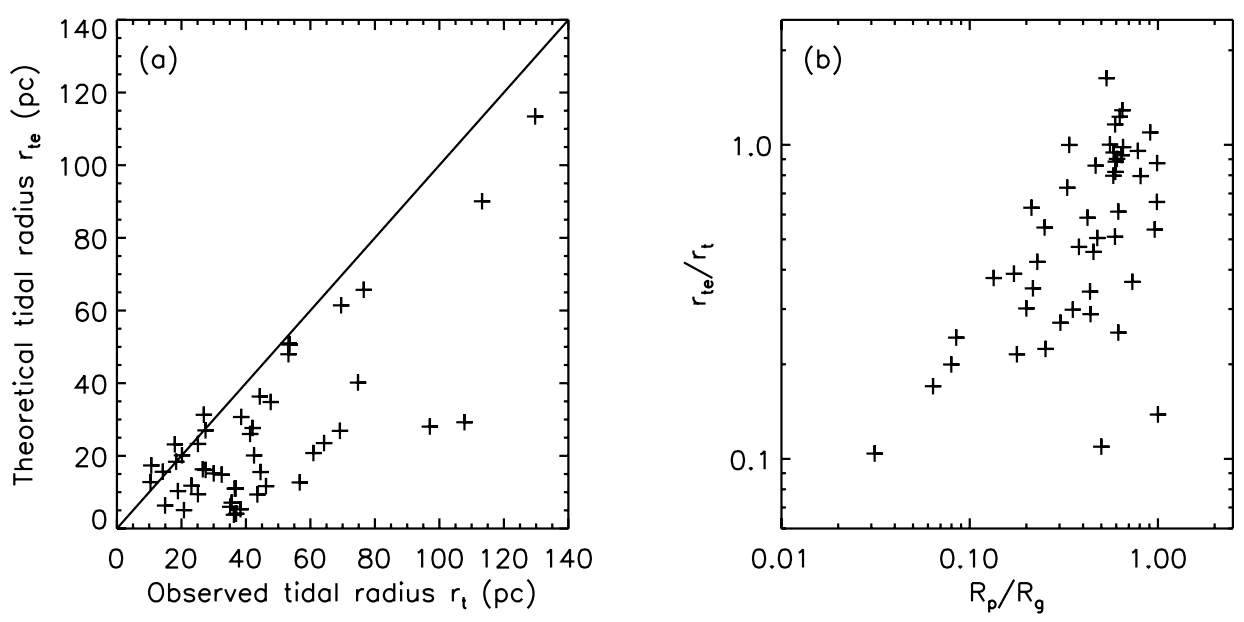

Figure 1. Panel a: Theoretical tidal radii $r_{t e}$ versus observed tidal radii $r_{t}$. Panel b:

Theoretical and observed tidal radii ratio $r_{t e} / r_{t}$ versus perigalactic and present distance ratio

$$
R_{p} / R_{g} \text {. }
$$

tidal radii calculated at the present positions $R_{g}$ of the clusters are also greater than the observed ones. At the apogalactic distances, computed values are consequently larger. Thus the tidal radius of a cluster is not only determined by the extent tidal field of the Galaxy when it orbits away from the perigalactic position, but is also affected by other dynamical processes.

\section{Orbital phase dependance of globular cluster's tidal radii}

Following Meziane \& Colin (1996), we also define the difference between the theoretical and observed tidal radii by introducing $r_{t e} / r_{t}$, and show this ratio between the perigalactic and present distances $R_{p} / R_{g}$ in panel b of Fig.1. A general trend appears to be in this figure, indicating the dependence of tidal radius on the orbital phase. When a cluster is near its perigalactic position, the theoretical tidal radius gives a good estimation of its observed value. The linear Pearson correlation coefficient of the logarithms between these two ratios is 0.74 , corresponding to a probability of correlation of $99.99999 \%$.

\section{References}

Hoerner, S. Von. 1957, ApJ, 125, 451

Innanen, K. A. $1983 A J, 88,338$

King, I. R. 1962, $A J, 67,471$

King, I. R. 1966, $A J, 71,64$

Meziane, K. \& Colin, J. 1996, A\& A, 306, 747

Trager, S. C., et al. 1993, ASP-CS, 50, 347

Wu, Z.-Y., Shu, C.-G., \& Chen, W.-P. 2003, Chin. Phys. Lett., 20, 1648

Wu, Z.-Y., Zhou, X., \& Ma, J. 2004, Chin. Phys. Lett., 21, 418 